\title{
Análise epidemiológica das pericardiotomias realizadas em um hospital universitário de Curitiba
}

\section{Epidemiological analysis of pericardiotomys held in a university hospital of Curitiba}

\author{
Márcia Mie Uchimura ${ }^{1}$; Juliana Battiston ${ }^{1}$; Patrícia Moreira ${ }^{2}$; Carla Martinez Menini Stahlschmidt, ACBC-PR: ${ }^{3}$; \\ FÁBIO LUIZ LUBACHEVSKI, AsCBC-PR ${ }^{4}$
}

\section{R E S U M O}

\begin{abstract}
Objetivo: Analisar epidemiologicamente a utilização da janela pericárdica(JP) no diagnóstico de lesão cardíaca em um hospital universitário de trauma de Curitiba. Métodos: Estudo observacional, retrospectivo, de análise dos prontuários de pacientes que foram submetidos a pericardiotomia por trauma contuso ou penetrante, no período de seis anos, no serviço de Urgência e Emergência do Hospital Universitário Cajuru. Resultados: 120 pacientes foram submetidos à Janela Pericárdica no período acima referido. A faixa etária variou de 15 a 80 anos, sendo a maior prevalência entre os 20 a 30 anos (49,7\%), 105(87,5\%) pacientes eram homens e $15(12,5 \%)$ mulheres. Os traumas fechados foram 14(11,67\%) e penetrantes 105(87,5\%). Dos penetrantes, 41 foram por ferida de arma branca, 60 por ferida de arma de fogo e quatro por ambas. Quanto à localização das lesões: 47,5\% foram precordiais, 34,16\% em transição tóraco-abdominal, 5,0\% em ambas e 13,33\% em outras localizações. Das JP realizadas, 72,5\% foram negativas e $27,5 \%$ positivas. Dentre as positivas, as lesões cardíacas encontradas foram: átrio direito $21,2 \%$, ventrículo direito $30,3 \%$, ventrículo esquerdo $24,2 \%$, aorta ascendente $3 \%$, nenhuma lesão $21,2 \%$. Houve 35 óbitos: 18 deles até 24 hs e 17 após 24hs. Conclusão: A janela pericárdica foi mais realizada em homens jovens com ferimentos penetrantes por arma de fogo, em sua maioria com lesão do ventrículo direito como principal achado, concordando com a literatura revisada.
\end{abstract}

Descritores: Janela pericárdica. Pericardiotomia. Trauma. Cirurgia.

\section{INTRODUÇÃO}

$\mathrm{O}$ trauma prevalece como a principal causa de morte entre adultos joven $\mathrm{s}^{1-3}$, em virtude do aumento da violência e do desenvolvimento de meios de transporte mais velozes ${ }^{1,3}$.

A maioria dos pacientes que sofre lesões cardíacas penetrantes não sobrevive até receber atendimento médico ${ }^{4-7}$. Esta lesão é uma causa freqüente de óbito, cerca de $60 \%{ }^{6}$ e deve ser rapidamente diagnosticada ${ }^{4,6}$. $\mathrm{O}$ paciente pode estar hemodinamicamente estável, porém, próximo a um colapso circulatório ${ }^{8}$. A clínica depende do tipo de lesão, do tempo entre a lesão e chegada ao centro de atendimento e de seu manejo inicial ${ }^{8}$.

Pacientes com tamponamento cardíaco ou em choque, que não respondem à reposição volêmica, necessitam de abordagem cirúrgica urgente 2,8-10. Já os hemodinamicamente estáveis, podem ser submetidos a exames não invasivos 2,6,8,10 como a tomografia computadorizada ${ }^{10}$ ou o FAST(focused assessment with sonography for trauma), que pode indicar a JP imediatamente ${ }^{6}$ se o resultado for questionável ${ }^{8}$ ou dispensá-la ${ }^{2}$.

A janela pericárdica é considerada por alguns autores ${ }^{4,7,9}$ o padrão-ouro para o diagnóstico de lesão car- díaca, pois rapidamente identifica ou exclui a presença de ferimento, tem alta sensibilidade e especificidade ${ }^{2,4,9,11}$, de fácil execução ${ }^{8}$. A visualização direta do pericárdio assegura se há sangue ou coágulo no saco pericárdico 2,4,11. Se o resultado da janela for negativo, com saída de líquido claro, a incisão cirúrgica pode ser fechada ${ }^{4,11,12}$. Se for positiva, há indicação absoluta de toracotomia ${ }^{7}$. Em casos nos quais a ecografia não estiver disponível ou o paciente apresentar indicação cirúrgica imediata, está indicada a JP4,11.

Quando o paciente apresenta lesões abdominais associadas, ele pode ser submetido a laparotomia exploradora com janela pericárdica transdiafragmática ${ }^{4}$.

O objetivo do estudo é analisar epidemiologicamente as pericardiotomias realizadas durante seis anos no Hospital Universitário Cajuru, avaliar os dados obtidos e relacioná-las ao trauma.

\section{MÉTODOS}

Foi realizado um estudo observacional, retrospectivo, longitudinal, através da análise de prontuários de pacientes submetidos à JP, no Hospital Universitário Cajuru, 
no período de 01 de janeiro de 2002 a 31 de dezembro de 2007.

Foram selecionados 120 prontuários. Os dados foram coletados utilizando-se um protocolo com os seguintes itens: idade, gênero, tipo de trauma, local da lesão, informações da ficha do Sistema Integrado de Atendimento ao Trauma em Emergência (SIATE) que é o serviço de atendimento pré-hospitalar ao traumatizado no Paraná) e da admissão hospitalar, reposição de cristalóides ou sangue, exames de imagem realizados, gasometria, motivo da janela pericárdica, resultado da JP e tipo da lesão cardíaca, transfusão intra-operatória, complicações pós-operatórias e óbito antes ou após 24 horas.

As informações obtidas foram processadas pelo programa Epi-info, versão 3.5.1, e analisadas.

O projeto foi previamente aprovado pelo Comitê de Ética em Pesquisa da Pontifícia Universidade Católica do Paraná.

\section{RESULTADOS}

O estudo compreendeu 120 pacientes, destacando-se o sexo masculino, representando 105 pacientes.

A faixa etária variou entre 15 a 80 anos, sendo a maior prevalência entre 20 a 30 anos (Tabela 1).

Das JP realizadas neste período, houve predomínio dos traumas penetrantes, sendo 105 (87,5\%) casos, $14(11,67 \%)$ por trauma fechado e um caso de pesquisa de neoplasia em paciente politraumatizado. Dos ferimentos penetrantes, 41 (39,05\%) foram por ferida de arma branca, $60(57,14 \%)$ por ferida de arma de fogo e quatro (3,81\%) por ambas (Tabela 2$) .47,5 \%$ das feridas estavam localizadas no precórdio, 34,16\% na transição tóraco-abdominal, 5,0\% em ambas e 13,33\% em outras localizações.

Os motivos que levaram à realização da janela pericárdica foram: o local da lesão próxima ao precórdio

Tabela 1 - Idade dos pacientes.

\begin{tabular}{lcc}
\hline Idade & $N^{\circ}$ pacientes & $\%$ \\
\hline$<20$ & 15 & 12,50 \\
$20-30$ & 59 & 49,17 \\
$30-40$ & 25 & 20,83 \\
$>40$ & 20 & 16,67 \\
Desconhecida & 1 & 0,83 \\
\hline
\end{tabular}

em 100 (83,33\%) pacientes, a instabilidade hemodinâmica trans ou pós-operatória sem causa evidente em seis (6\%) pacientes e $14(11,67 \%)$ por ambos os motivos.

Das JP realizadas, 87 (72,5\%) foram negativas e $33(27,5 \%)$ positivas. Dentre as positivas, as lesões cardíacas encontradas foram em ventrículo direito(VD), ventrículo esquerdo (VE), átrio direito(AD), aorta ascendente (Tabela 3). Observou-se que $32,8 \%$ das lesões no precórdio e $22,22 \%$ das que acometem a região de transição tóracoabdominal resultaram em janela positiva.

Houve 35(29\%) óbitos, 18(51\%) deles até 24 horas e 17 (49\%) após as 24 horas. De todos os óbitos, 15 $(42,8 \%)$ deles tiveram JP positiva e 27 (77\%) tiveram lesões associadas e 28 precisaram de transfusão intra-operatórias. Entre os casos fatais com lesão cardíaca, houve predomínio de injúria em VE e AD (Tabela 4).

A maioria dos pacientes evoluiu bem, sendo que 32 apresentaram complicações durante o internamento pósoperatório: três pacientes apresentaram coagulação intravascular disseminada, sete, síndrome da angústia respiratória aguda, , 10 infecções pulmonares, três, infecções no sítio cirúrgico e outras complicações não incluídas no protocolo de investigação.. Não houve nenhuma infecção ocasionada pelo cateter.

\section{DISCUSSÃO}

Os traumas torácicos representam uma causa importante de atendimento nos serviços de Emergência ${ }^{12,13}$. Acomete na maioria homens, jovens, que estão expostos a mecanismos de violência e agressão ${ }^{13,14}$, o que condiz com os dados coletados neste estudo.

De acordo com a literatura, a maioria dos traumas cardíacos é penetrante $2,3,14$, principalmente por arma de fogo ${ }^{2,14}$, sendo que as lesões penetrantes são as mais fatais $^{3,7}$, concordando com os dados encontrados neste estudo. O número de traumas penetrantes é elevado(87,5\%) em relação ao de traumas fechados $(11,67 \%)$.

Os traumas contusos podem lesar as câmaras cardíacas através das desacelerações bruscas ${ }^{3}$. A mortalidade nestes casos pode alcançar 71,4\% ${ }^{3}$. Fraga GP et al. notaram que o ventrículo esquerdo tende a ser mais acometido que o direito no trauma fechado. A alta pressão intracavitária e a baixa complacência do VE favorecem a ruptura por trauma fechado. Na investigação, houve lesão apenas de AD por causa contusa nos pacientes atendidos

Tabela 2 - Resultados das Janelas Pericárdicas.

\begin{tabular}{|c|c|c|c|c|c|c|c|}
\hline & \multicolumn{3}{|c|}{ Aberto } & \multicolumn{4}{|c|}{ Fechado } \\
\hline & FAB & FAF & $F A B$ e $F A F$ & atropelamento & colisão & $\begin{array}{l}\text { acidente } \\
\text { veiculo motor }\end{array}$ & queda \\
\hline JP positiva & 14 & 14 & 1 & 0 & 2 & 1 & 1 \\
\hline JP negativa & 27 & 46 & 3 & 2 & 3 & 3 & 2 \\
\hline Total & 105 & & & 14 & & & \\
\hline
\end{tabular}


Tabela 3 - Local das lesões.

\begin{tabular}{lcc}
\hline Local & Número & $\%$ \\
\hline Ventrículo Direito & 10 & 30,3 \\
Ventrículo Esquerdo & 8 & 24,2 \\
Átrio Direito & 7 & 21,2 \\
Aorta ascendente & 1 & 3 \\
Nenhuma lesão & 7 & 21,2 \\
\hline
\end{tabular}

Tabela 4 - Relação entre lesão cardíaca e óbitos.

\begin{tabular}{lcc}
\hline Lesão & Óbito antes de $24 \mathrm{~h}$. & Óbito após $24 \mathrm{~h}$. \\
\hline VE & 3 & 1 \\
VD & 2 & 2 \\
Aorta ascendente & 1 & 0 \\
$A D$ & 4 & 0 \\
\hline
\end{tabular}

pelo hospital, o que pode indicar a gravidade e letalidade pré-hospitalar de rotura cardíaca contusa. Já nas lesões penetrantes, houve predomínio de lesões de VD, concordando com alguns trabalhos ${ }^{3,11}$.

As lesões por arma de fogo causam grandes lacerações que provocam sangramento o qual pode não ser contido pelo saco pericárdico, levando rapidamente ao choque hemorrágico ${ }^{3}$. Os sinais clínicos relacionados à lesão cardíaca são: estado de choque hemorrágico, tamponamento cardíaco, alterações da ausculta cardía$\mathrm{ca}^{14,15}$. No Serviço do Hospital Cajuru, observou-se que a maioria dos pacientes investigados, com prontuário preenchido, apresentava-se clinicamente bem, com bulhas cardíacas normofonéticas e com pulsos periféricos palpáveis.

Os ferimentos cardíacos podem ser investigados de acordo com o protocolo e disponibilidade de equipamento de cada hospital. O FAST é um dos exames reco- mendados para pesquisa de lesão tanto em pacientes estáveis como instáveis pois tem sensibilidade aproximada de $100 \%$, especificidade em torno de $96,9 \%$ e acurácia de $97,3 \%{ }^{7}$.Tem a desvantagem de ser operador dependen$t^{2}$. Nos 120 pacientes investigados, nenhum deles foi submetido ao FAST por falta de condições propícias na sala de politraumatizados. Os critérios adotados para a indicação cirúrgica foram a instabilidade hemodinâmica e a localização da ferida próxima ao precórdio.

A JP, por sua sensibilidade e eficácia, é considerada o padrão-ouro e tem indicação em casos de suspeita de lesão cardíaca ${ }^{2,11,14}$. Por sua alta sensibilidade, deve-se ter cuidado na sua realização, qualquer descuido técnico pode resultar em falso-positivo. Pode ser encontrado sangue no saco pericárdico sem lesão cardíaca ${ }^{4,9}$. Verificou-se que em $21,2 \%$ das pericardiotomias positivas investigadas foram falso-positivo ${ }^{4,9}$. A positividade das janelas varia de acordo com o serviço onde é realizada. O resultado obtido no Hospital Cajuru foi de $27,5 \%$, e se enquadra dentro dos valores encontrados na literatura, em torno de 18 a $30 \%{ }^{4}$.

As duas abordagens de JP, subxifóidea e a transdiafragmática, têm bons resultados com alta sensibilidade e especificidade. $\mathrm{O}$ acesso abdominal necessita de maior atenção devido à possibilidade de contaminação pelo conteúdo da cavidade peritoneal ${ }^{9}$. A maioria das JP avaliadas neste estudo fez-se com acesso pela via transdiafragmática, utilizada de rotina no Serviço de Trauma do Hospital Cajuru..

Com o presente estudo, pode se concluir que, a janela pericárdica foi mais realizada em homens jovens com ferimentos penetrantes por arma de fogo, em sua maioria com lesão do ventrículo direito como principal achado. procedimento permite rápido diagnóstico, é indicado para casos em que há a suspeita de lesão cardíaca mas não é indispensável que o paciente apresente clínica. Se houver instabilidade hemodinâmica, a janela deve ser realizada prontamente; se o paciente permanecer estável é possível aguardar pelos exames de imagem.

\section{A B S T T R A C}

Objective: Epidemiologically analyze the use of the pericardiostomy in the diagnosis of cardiac injury in a trauma universitary hospital in Curitiba. Methods: Observacional, retrospective study, analyzing medical records of patients who were submitted to penetrating or blunt trauma, in a period of 06 years, in Urgency and Emergency unit of Cajuru Universitary Hospital. Results: 120 patients had been submitted to pericardiostomy in the period above related. The age group varied from 15 to 80 years, the major prevalence between 20 and 30 years (49.7\%), 105 patients were men and 15 women. Blunt trauma corresponded to 14 patients and penetrating to 105 patients. From penetrating ones, 41 patients were inflicted by stabwound, 60 by gunshot wound and 4 by both. About injury sites: $47.5 \%$ had been precordial, $34.16 \%$ in thoraco-abdominal transistion, $5.0 \%$ in both and $13.33 \%$ in other sites. From the accomplished JP, $72.5 \%$ had been negative and $27.5 \%$ positive. Among the positive, cardiac injuries diagnosed by immediate thoracotomy were: right atrium $21.2 \%$, right ventricle $30.3 \%$, left ventricle $24,2 \%$, ascending aorta 3\%, no injury $21.2 \%$. 35 patients died: 18 of them up to $24 \mathrm{hs}$ and 17 after 24hs. Conclusion: The pericardiostomy were performed mostly in young men with penetrating trauma caused by gunshot wounds., in its majority with injury of the right ventricle as main finding, which agreed to the reviwed literature.

Key words: Pericardiostomy. Pericardiotomy. Trauma. Surgery. 


\section{REFERENCIAS}

1. Cuba RMBF, Bezerra JAF. Traumatismo torácico. Estudo retrospectivo de 168 casos. Rev Col Bras Cir. 2005; 32(2):57-9.

2. Gabrielli MN, Muñoz SG, Troncoso PG, Felmer OE, Kinast AC, Sánchez $\mathrm{AH}$ et al. Herida penetrante cardíaca. Cuad Cir. 2007; 21(1):75-83.

3. Fraga GP, Heinzl LR, Longhi BS, Silva DC, Fernandes Neto FA, Mantovani M. Trauma cardíaco: estudo de necropsias. Rev Col Bras Cir. 2004; 31(6):386-90.

4. Mantovani M, Espinola JP, Fraga GP. Janela pericárdica transdiafragmática no diagnóstico de Lesão cardíaca. Rev Col Bras Cir. 2006; 33(1):29-34.

5. Cáceres M, Buechter K, Rodriguez JA, Liu D. Delayed hemopericardium after penetrating chest trauma: thoracoscopic pericardial window as a therapeutic option. South Med J. 2004; 97(10):994-8

6. Barbelen A, Huerta S, Mendonza R, Patel CV. Left ventricle injury with a normal pericardial window: case report and review of the literature. J Trauma. 2007;63(2):414-6.

7. Navsaria $\mathrm{PH}, \mathrm{Nicol}$ AJ. Haemopericardium in stable patients after penetrating injury: Is subxiphoid pericardial window and drainage enough? A prospective study. Injury. 2005; 36(6):745-50. Epub 2005 Mar 128.

8. Méndez EAJ, Zamora JL, Zeledón FS, Zamora FR. Trauma cardíaco: una revisión práctica II Parte. Traumatismo penetrante. Rev Costarric Cardiol. 2005; 7(1):39-46.

9. Fraga GP, Espinola JP, Mantovani M. Pericardial window used in the diagnosis of cardiac injury. Acta Cir Bras. 2008; 23(1):208-15.

10. Stassen NA, Lukan JK, Spain DA, Miller FB, Carrillo EH, Richardson JD, Battistella FD. Reevaluation of diagnostic procedures for transmediastinal gunshot wounds. J Trauma. 2002; 53(4):635-8; discussion 638.

11. Isla Ortiz D, Barrera F, Espinosa Mendonza A. Ventana pericárdica en pacientes com herida penetrante em tórax hemodinámicante estables. Trauma. 2004; 7(3):85-8.

12. Meredith JW, Hoth JJ. Thoracic trauma: when and how to intervene. Surg Clin North America. 2007; 87(1):95-118.

13. Valenzuela MD, Cancin PA, Cabezas FS, Danoso GD, La Torre I. Experiencia en traumatismo torácico: Hospital Valparaíso. Rev Chil Cir. 2003; 55(5):449-53.

14. Rodriguez-Ortega MF, Cárdenas-Martínez G, Vázquez Minero JC, Gómez García MA, Veja Rivera F, Zepeda Sanabria JR, et al. Experiencia en el tratamiento de lesiones de corazón por el cirujano general. Cir Gen. 2004; 26(1):7-12.

15. Giurgius M, Al Asfar F, Dhar PM, Al Awadi N. Penetrating cardiac Injury. Med Princ Pract. 2006; 15(1):80-2.

Recebido em 28/01/2009

Aceito para publicação em 30/03/2009

Conflito de interesse: nenhum

Fonte de financiamento: nenhuma

\section{Como citar esse artigo:}

Uchimura MM, Battiston J, Moreira P, Stahlschmidt CMM, Lubachevski FL. Análise epidemiológica das pericardiotomias realizadas em um hospital universitário de Curitiba. Rev Col Bras Cir. [periódico na Internet] 2010; 37(2). Disponível em URL: http://www.scielo.br/rcbc

Endereço para correspondência:

Márcia Mie Uchimura

E-mail: marcia_uchimura@hotmail.com 